\title{
Effets respectifs du changement de l'alimentation et de l'environnement lors de la sortie au pâturage sur la composition chimique et l'aptitude à la coagulation du lait
}

\author{
JB Coulon 1 \\ avec la collaboration technique d'E Albaret ${ }^{2}$ et M Jaworek ${ }^{2}$ \\ 1 INRA-Theix, laboratoire Adaptation des herbivores aux milieux, 63122 Saint-Genès-Champanelle; \\ 2 INRA, domaine expérimental de La Borie, 15190 Marcenat, France
}

(Reçu le 8 juin 1994 ; accepté le 9 août 1994)

\begin{abstract}
Résumé - Seize vaches Tarentaises multipares en milieu de lactation ont reçu à l'auge pendant 3 sem une ration composée de regain de prairie naturelle (lot $R$ ) ou d'herbe verte (lot $H$ ), distribuée de manière isoénergétique (respectivement 10,7 et 10,6 UFL/j). Les 2 lots ont ensuite été mis à l'herbe. La nature de la ration n'a eu d'effet ni sur la production de lait, ni sur sa composition chimique, ni sur son aptitude à la coagulation. À la mise à l'herbe, dans les 2 lots, la production laitière et le $\mathrm{pH}$ du lait ont augmenté fortement (respectivement de $1,8 \mathrm{~kg} / \mathrm{j}$ et 0,04 unité, $\mathrm{P}<0,01$ ). Le taux protéique a augmenté plus fortement dans le lot $\mathrm{H}$ que dans le lot $\mathrm{R}$ (respectivement $+2,8 \mathrm{et}+1,3 \mathrm{~g} / \mathrm{kg}, P<0,05$ ). Ces modifications ont été associées à des variations importantes du temps de coagulation du lait mesuré à pH initial $(+1,5 \mathrm{~min}, P<0,01)$ et de la fermeté du gel mesurée à $\mathrm{pH}$ standardisé (respectivement $+1,5$ et $+3,2 \mathrm{~mm}$ pour les lots $R$ et $H, P<0,01$ ). Ces modifications sont essentiellement à relier à l'augmentation des apports alimentaires consécutifs à la mise à l'herbe.
\end{abstract}

vache laitière / mise à l'herbe / coagulation du lait

Summary - Effects of feeding and environmental changes during turn out to pasture on cows' milk composition and coagulation properties. Sixteen Tarentaise dainy cows in mid lactation received during 3 wk a ration of aftermath (group R) or grass (group H). The quantities of each forage were adjusted to allow the same energy supply. The 2 groups were then turned out to pasture on the same paddock. During the 3 weeks indoors, there was no difference between the 2 groups in milk yield (10.9 and $11.1 \mathrm{~kg} / \mathrm{d}$, respectively in groups $R$ and $\mathrm{H}$ ), fat content (38.3 and $38.6 \mathrm{~g} / \mathrm{kg}$, respectively), protein content ( 31.7 and $31.2 \mathrm{~g} / \mathrm{kg}$, respectively) and milk coagulation properties. At turn out to pasture, milk yield and $\mathrm{pH}$ increased greatly in the 2 groups $(+1.8 \mathrm{~kg} / \mathrm{d}$ and +0.04 unit, respectively, $\mathrm{P}<0.01)$. Protein content increased more greatly in group $H$ than in group $R(+2.8$ and $+1.3 \mathrm{~g} / \mathrm{kg}$, respectively, 
$\mathrm{P}<0.05)$. As casein and soluble proteins contents increased in the same proportion, casein/protein ratio was not affected by turn out to pasture. In the 2 groups, turning out to pasture induced a significant $\mathbb{P}<$ 0.01 ) increase of $1.5 \mathrm{~min}$ in milk rennet clotting time (measured at the milk natural $\mathrm{pH}$ ). Curd firmness (measured after pH standardization at 6.60) increased more $(\mathrm{P}<0.01)$ in group $\mathrm{H}(+3.2 \mathrm{~mm})$ than in group $R(+1.5 \mathrm{~mm})$. These variations result mainly from the increase of the nutritive supplies at turn out to pasture.

dairy cow / turn out to pasture / coagulation properties

\section{INTRODUCTION}

La mise à l'herbe est une période délicate pour les vaches laitières. Elle s'accompagne de modifications de l'alimentation et de l'environnement importantes et parfois brutales, en particulier lorsque les animaux sont hivernés en stabulation entravée et sortent rapidement au pâturage. De nombreuses études ont été entreprises pour décrire l'évolution de la production et de la composition chimique du lait à cette période. En règle générale, la production de lait augmente ainsi que sa richesse en protéines (Rook et al, 1960 ; Demarquilly et Journet, 1962 ; Decaen et Journet, 1966 ; Vignon et al, 1978 ; Rearte et al, 1986 ; Coulon et al, 1986 et 1988 ; Dubeuf et al, 1991). L'évolution de la teneur en matières grasses est plus variable et dépend en particulier de son niveau initial et de la nature et du stade de l'herbe pâturée (Hoden et al, 1985). La composition des matières grasses et des matières protéiques est aussi modifiée (Rook et al, 1960 ; Decaen et Ghadaki, 1970 ; Vignon et al, 1978 ; Coulon et al, 1988). Ces modifications dépendent cependant de nombreux facteurs et en particulier du type de ration hivernale, du niveau des apports nutritifs correspondants (Rook et al, 1960 ; Le Du et al, 1979 ; Hoden et al, 1985 ; Coulon et al, 1986), du mode de transition alimentaire (Coulon et al, 1988) et de la conduite du pâturage.

Les modifications de l'aptitude à la coagulation du lait à la mise à l'herbe ont fait l'objet de moins de travaux (Chapman et Burnett, 1972 ; Grandison et al, 1984 ;
Mariani et al, 1985 ; Vertès et al, 1989 ; Macheboeuf et al, 1993). La plupart ont mis en évidence une amélioration importante du temps de raffermissement et de la fermeté du gel, en grande partie expliquée par l'augmentation parallèle du taux de caséines du lait. Le $\mathrm{pH}$ du lait augmente parfois de manière importante mais le temps de coagulation, mesuré à $\mathrm{pH}$ standardisé, est en général peu modifié.

Aucun de ces travaux ne permet cependant de faire la part entre l'effet propre du changement de nature de ration, celui des modifications du niveau des apports nutritifs associés, et celui de l'environnement. L'objectif de cette étude a été de dissocier les effets respectifs de ces différents facteurs sur la composition chimique du lait et son aptitude à la coagulation.

\section{MATÉRIEL ET MÉTHODES}

\section{Animaux}

Seize vaches Tarentaises multipares en milieu de lactation ont été utilisées. En début d'essai, elles étaient en moyenne en $20^{\circ}$ semaine de lactation et produisaient $11,5 \mathrm{~kg} / \mathrm{j}$ de lait. Au cours d'une période préexpérimentale de 4 sem ( $P 1$, 20 avril au 17 mai), tous les animaux ont reçu une ration composée de regain offert à raison de $12,5 \mathrm{~kg} \mathrm{MS} / \mathrm{j}$. Ce fourrage provenait d'une parcelle de prairie permanente composée, sur la base de la MS, de $85 \%$ de Graminées (pâturin, ray-grass anglais, dactyle) et de $15 \%$ d'autres plantes (pissenlit, rumex, plantain, renoncule). Trois lots ont ensuite été constitués, sur la base de la date de vêlage et de la production et de la 
composition chimique du lait observée en période préexpérimentale. Pendant 3 sem (P2) le premier lot $(R)$ a continué de recevoir la même ration, tandis que les 2 autres lots recevaient, à l'auge, de l'herbe coupée, en quantités limitées (de manière à apporter la même quantité d'énergie que dans le lot $R$ ) pour l'un et à volonté pour l'autre. Cette herbe provenait d'une parcelle de prairie permanente composée, sur la base de la MS, de $75 \%$ de Graminées (ray-grass anglais, pâturin, dactyle, folle avoine), de $15 \%$ de pissenlits et de $10 \%$ d'autres plantes (renoncule, plantain). Compte tenu des conditions climatiques qui ont conduit à des teneurs en matière sèche très faibles de l'herbe (12 à 15\%), les quantitès ingérées par le lot recevant l'herbe à volonté ont été très peu différentes de celles du lot recevant l'herbe en quantités limitées. Ces 2 lots ont donc été regroupées pour l'analyse des résultats (lot $\mathrm{H}$ ). Le 8 juin, tous les animaux ont été sortis au pâturage pendant 5 sem (P3), sur une parcelle de prairie permanente différente de celle ayant fourni l'herbe au cours de la période 1 , mais de composition floristique voisine : $85 \%$ de Graminées (ray grass anglais, dactyle et fétuque), $5 \%$ de Légumineuses et $10 \%$ d'autres plantes.

Compte tenu des apports nutritifs permis par les fourrages (correspondant à la production d'environ $13 \mathrm{~kg}$ de lait à $4 \%$ de matières grasses) et du faible niveau de production des animaux en début d'essai, seuls 4 d'entre eux ont reçu, au cours des périodes 1 et 2 , un apport d'orge, compris entre 0,5 et $2 \mathrm{~kg} / \mathrm{j}$, de manière à ce que leurs besoins énergétiques soient couverts. Un complément minéral ( $8 \mathrm{P}-8 \mathrm{Ca}-10 \mathrm{Mg})$ a été distribué à tous les animaux durant tout l'essai à raison de $200 \mathrm{~g} / \mathrm{j}$.

Tableau I. Caractéristiques des aliments utilisés.

\section{Mesures et analyses}

Au cours des 2 premières périodes, les quantités ingérées de fourrage et de concentré ont été mesurées tous les jours. La composition chimique (matière organique, matières azotées totales, cellulose brute, calcium et phosphore) du regain a été analysée une fois dans l'essai. La composition chimique et la caractérisation de la composition floristique de l'herbe ont été réalisées chaque semaine pendant la période 2 et durant les 2 pre mières semaines de la période 3 . La composition en acides aminées du regain et de l'herbe (périodes 2 et 3 ) a été déterminée à l'aide de la méthode décrite par Le Hénaff (1991). Les caractéristiques des aliments utilisés sont précisées au tableau I

La production laitière a été mesurée individuellement tous les jours. Les taux butyreux et protéique ont été mesurés 2 fois par semaine au cours des 3 premières semaines de la période 1 et des 4 dernières semaines de la période 3 . Ils ont été mesurés 5 j par semaine durant le reste de l'essai. Les animaux ont été pesés une fois par semaine.

Un échantillon de lait de $50 \mathrm{ml}$ a été prélevé, pour chaque vache, à la traite du matin, deux j par semaine au cours de la dernière semaine de la première période, durant chaque semaine de la deuxième période et au cours des 2 premières semaines de la troisième période. Sur cet échantillon, le $\mathrm{pH}$ a été mesuré (à une température stabilisée à $20^{\circ} \mathrm{C}$ ), ainsi que les caractéristiques rhéologiques du lait (temps de coagulation, temps de raffermissement et fermeté du gel à $30 \mathrm{~min}$ ) à l'aide d'un appareil Formagraph, selon la méthode proposée par Mc Mahon et Brown (1982). Ces

\begin{tabular}{|c|c|c|c|c|c|c|c|c|c|c|}
\hline $\begin{array}{l}\text { MS } \\
(\%)\end{array}$ & $M O$ & $C B$ & MAT & $\mathrm{Ca}$ & $P$ & $\begin{array}{c}U F L \\
(k g M S)\end{array}$ & $\begin{array}{l}\text { PDIN } \\
(g / k g\end{array}$ & PDIE & $1 \%$ & $\begin{array}{r}M e \\
D I E)\end{array}$ \\
\hline 91 & 906 & 270 & 157 & 0,46 & 0,34 & 0,82 & 98 & 89 & & \\
\hline 14,5 & 918 & 291 & 153 & 0,47 & 0,33 & 0,91 & 96 & 84 & 7,2 & \\
\hline 14,6 & 909 & 269 & 248 & nd & nd & 0,88 & 93 & 86 & 7,2 & \\
\hline
\end{tabular}

a Moyenne des 2 dernières semaines de la période 2 ; b première semaine de la période 3. 
mesures ont été réalisées à $\mathrm{pH}$ initial et à $\mathrm{pH}$ standardisé à 6,60 . Toutes les mesures ont été réalisées en double. Une fois par semaine sur les mêmes échantillons les teneurs en protéines solubles et en caséines (Rowlands, 1938), en urée (Moore et Sax, 1965), en protéines totales et en lactose (méthode infra-rouge), et en calcium (IOS, 1987) du lait ont été mesurées.

L'identification des variants génétiques de la caséine kappa a été réalisée sur des échantillons de lait, par isoélectrofocalisation selon la méthode proposée par Seibert et al (1985). Dix vaches étaient de type $A A$ et 6 de type $A B$.

\section{Analyses statistiques}

L'effet de la nature du fourrage a été apprécié sur les valeurs moyennes des 2 dernières semaines de la période 2, par analyse de variance. Les facteurs introduits dans l'analyse ont été le lot et le variant de la caséine kappa. $\mathrm{Ce}$ dernier facteur a été introduit en raison de son effet important sur les caractéristiques rhéologiques du lait (Grosclaude, 1988 ; Machebœuf et al, 1993). La valeur de la variable au cours des 2 dernières semaines de la période 1 a été introduite en covariable dans l'analyse.

L'effet de la sortie au pâturage a été appréciée par l'étude de l'écart entre les valeurs observées en dernière semaine de la période 2 et en première semaine de la période 3 . Les facteurs introduits dans l'analyse ont été le lot et le variant de la caséine kappa.

\section{RÉSULTATS}

\section{Effet de la nature de l'alimentation}

Comme prévu par le protocole, les apports énergétiques ont été identiques dans les 2 lots. Les apports azotés ont été supérieurs dans le lot $R$ (respectivement +139 g/j PDIN et $+168 \mathrm{~g} / \mathrm{j}$ PDIE), en raison du rapport $P D I / U F L$ plus élevé du regain comparativement à l'herbe (120 g PDIN/UFL et $109 \mathrm{~g}$ PDIE/UFL pour le regain contre respectivement 105 et 92 pour l'herbe). Dans les 2 lots les bilans énergétiques et azotés ont été largement positifs (tableau II).

La nature de la ration n'a eu d'effet significatif ni sur la production laitière (respectivement 10,9 et $11,1 \mathrm{~kg} / \mathrm{j}$ pour les lots $R$ et $H$ ) ni sur la teneur en matières grasses $(38,3$ contre $38,6 \mathrm{~g} / \mathrm{kg})$ ou en protéines $(31,7$ contre $31,2 \mathrm{~g} / \mathrm{kg}$ ). Les teneurs en lactose, en caséines, en protéines solubles, en urée et en calcium du lait ont aussi été identiques dans les 2 lots (tableau II).

Le pH du lait a été supérieur (de 0,04 unités, $P<0,05$ ) dans le lot $\mathrm{R}$, de même que le temps de coagulation du lait mesuré à $\mathrm{pH}$ initial $(+1,4 \mathrm{~min}, P<0,01)$. Mesuré à $\mathrm{pH}$ standardisé, cette différence disparaît. Le temps de raffermissement et la fermeté du gel n'ont pas été différents, à pH initial ou à $\mathrm{pH}$ standardisé, entre les 2 lots (tableau II).

\section{Effet de la sortie au pâturage}

À la sortie au pâturage, tous les paramètres de composition du lait, à l'exception du taux butyreux, ont été significativement modifiés, souvent de manière plus sensible dans le lot $\mathrm{H}$ que dans le lot $\mathrm{R}$ (tableau III). La production laitière a augmenté de manière voisine dans les 2 lots $(+1,8 \mathrm{~kg} / \mathrm{j}$ en moyenne, $P<0,01)$, et le taux de lactose a diminué de $1,5 \mathrm{~g} / \mathrm{kg}(P<0,01)$. Le taux protéique a augmenté plus fortement $(P<0,05)$ dans le lot $\mathrm{H}(+2,8 \mathrm{~g} / \mathrm{kg})$ que dans le lot $\mathrm{R}$ $(+1,3 \mathrm{~g} / \mathrm{kg})$. Cette augmentation est due à la fois à celle du taux de caséines (respectivement $+1,1$ et $+2,2 \mathrm{~g} / \mathrm{kg}$ ) et à celle du taux de protéines solubles (respectivement $+0,3$ et $+0,6 \mathrm{~g} / \mathrm{kg}$ ), de sorte que le rapport caséines/protéines est resté identique au cours des 2 périodes. Dans le lot $R$, les taux d'urée et de calcium ont été légèrement modifiés à la sortie au pâturage (respectivement $-0,06 \mathrm{~g} / \mathrm{l}$ sérum et $+0,1 \mathrm{~g} / \mathrm{kg}$, $P<0,05)$. 
Tableau II. Effet de la nature de la ration sur les performances des animaux.

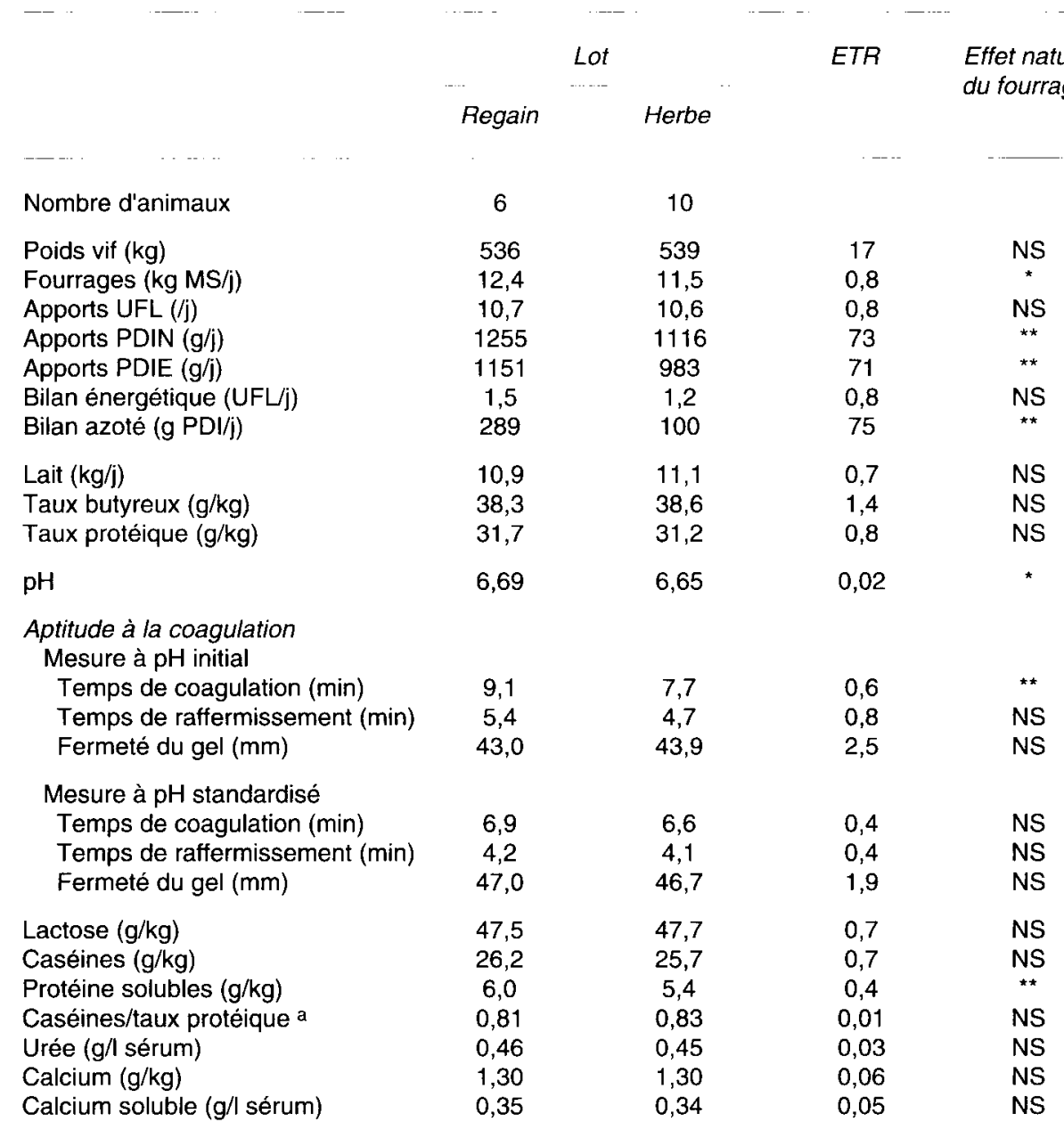

a Le taux protéique utilisé pour calculer ce rapport est celui mesuré sur l'échantillon ayant servi à la mesure du taux de caséines du lait. II est légèrement différent de celui présenté en début de tableau, qui représente la moyenne des analyses effectuées sur la période considérée. * $P<0,05 ;{ }^{\star \star} P<0,01$.

Le $\mathrm{pH}$ du lait a augmenté dans les 2 lots $(+0,04$ unités en moyenne, $P<0,01)$, de même que le temps de coagulation à $\mathrm{pH}$ initial $(+1,5 \mathrm{~min}$ en moyenne, $P<0,01)$. Mesuré à pH standardisé, le temps de coagulation n'a pas été modifié par la sortie au pâturage. La vitesse de raffermissement et la fermeté du gel ont été améliorées de manière plus importantes dans le lot $\mathrm{H}$ que dans le lot $R$, que la mesure ait été réalisée à $\mathrm{pH}$ intial ou à $\mathrm{pH}$ standardisé (par exemple, $+6,3 \mathrm{~mm}$ de fermeté du gel dans le lot $\mathrm{H}$ contre $+2,9 \mathrm{~mm}$ dans le lot $\mathrm{R}$, $P<0,01)$. 
Tableau III. Effet de la sortie au pâturage sur les performances des animaux : différence entre la première semaine au pâturage et la dernière semaine à l'étable, selon la ration offerte à l'étable.

\begin{tabular}{|c|c|c|c|c|}
\hline Lot & Regain & Herbe & ETR & $\begin{array}{l}\text { Signification } \\
\text { statistique a }\end{array}$ \\
\hline & & & & -- \\
\hline$n$ & 6 & 10 & & \\
\hline Lait $(\mathrm{kg} / \mathrm{j})$ & $1,7^{\star \star}$ & $1,9^{\star \star}$ & 0,8 & NS \\
\hline Taux butyreux (g/kg) & 0,2 & $-1,3$ & 1,8 & NS \\
\hline Taux protéique $(\mathrm{g} / \mathrm{kg})$ & $1,3^{* *}$ & $2,8^{\star \star}$ & 1,0 & * \\
\hline $\mathrm{pH}$ & $0,03^{*}$ & $0,04^{* *}$ & 0,03 & NS \\
\hline \multicolumn{5}{|l|}{$\begin{array}{l}\text { Aptitude à la coagulation } \\
\text { Mesure à } \mathrm{pH} \text { initial }\end{array}$} \\
\hline Temps de coagulation (min) & $1,4^{\star *}$ & $1,6^{\star \star}$ & 0,8 & NS \\
\hline Temps de raffermissement (min) & 0,2 & $-0,4^{\star}$ & 0,6 & NS \\
\hline Fermeté du gel $(\mathrm{mm})$ & 0,2 & $4,2^{\star \star}$ & 1,7 & $\star \star$ \\
\hline \multicolumn{5}{|l|}{ Mesure à pH standardisé } \\
\hline Temps de coagulation (min) & $-0,4^{*}$ & $-0,2$ & 0,4 & NS \\
\hline Temps de raffermissement (min) & $-0,4^{\star}$ & $-1,1^{\star \star}$ & 0,4 & * \\
\hline Fermeté du gel $(\mathrm{mm})$ & $1,5^{* *}$ & $3,2^{\star *}$ & 0,5 & $\star \star$ \\
\hline Lactose $(\mathrm{g} / \mathrm{kg})$ & $-1,3^{\star}$ & $-1,6^{\star \star}$ & 1,1 & NS \\
\hline Caséines $(\mathrm{g} / \mathrm{kg})$ & $1,1^{*}$ & $2,2^{\star \star}$ & 1,0 & $\star$ \\
\hline Protéines solubles ( $\mathrm{g} / \mathrm{kg}$ ) & 0,3 & $0,6^{\star}$ & 0,6 & * \\
\hline Caséines/taux protéique & 0,00 & 0,00 & 0,02 & NS \\
\hline Urée ( $g / /$ sérum) & $-0,06^{\star}$ & 0,00 & 0,02 & NS \\
\hline Calcium $(\mathrm{g} / \mathrm{kg})$ & $0,1^{*}$ & 0,05 & 0,09 & NS \\
\hline Calcium soluble ( $g / 1$ sérum) & $0,06^{\star \star}$ & $0,03^{\star}$ & 0,03 & NS \\
\hline
\end{tabular}

a Signification statistique de la différence entre les 2 lots, c'est-à-dire de l'interaction entre la nature du fourrage distribué à l'auge et la mise à l'herbe. Les écarts suivis d'un symbole sont significativement différents de 0 ; ${ }^{\star} P<0,05 ;{ }^{\star *} P<0,01$.

\section{DISCUSSION ET CONCLUSION}

Cette étude a confirmé les effets favorables de la sortie au pâturage, à la fois sur la production de lait et sa teneur en protéines (Hoden et al, 1985) et son aptitude à la coagulation (Vertès et al, 1989 ; Macheboeuf et al, 1993). Ces effets ont vraisemblablement été amplifiés par le faible niveau de besoins des animaux utilisés. En effet, chez des animaux bien alimentés au cours de l'hiver, l'effet de la mise à l'herbe est d'autant moins important que leur niveau de production est plus élevé (Coulon et al, 1986 et 1988).
Cette étude montre que ces effets ne sont pas liés au changement de nature de la ration : à même niveau d'apport énergétique, une herbe verte et un regain de composition floristique et chimique voisine conduisent à des performances de production et de composition du lait identiques. Ces observations vont dans le sens de celles de Vérité et Journet (1970) sur de l'herbe verte ou déshydratée. Lorsque des différences sont observées entre une herbe verte distribuée à l'auge et des fourrages conservés (Decaen et Journet, 1966 ; Decaen et Ghadaki, 1970), elles sont dues 
en grande partie à des niveaux d'apports nutritifs différents, liés à la distribution à volonté des fourrages.

L'essentiel des modifications observées ont eu lieu lorsque les animaux ont été sortis au pâturage. L'amélioration de la production laitière et du taux protéique peut d'abord être expliquée par une augmentation importante des apports nutritifs, et en particulier énergétiques, dont l'influence sur la production laitière et le taux protéique du lait est connue, même lorsque les besoins sont couverts (Coulon et Rémond, 1991). Compte tenu des caractéristiques de l'herbe offerte au pâturage, l'augmentation des apports énergétiques peut être estimée à environ 2 UFL/j par rapport aux rations distribuées à l'auge (Coulon et Faverdin, 1994), ce qui permet d'expliquer l'essentiel de l'augmentation de production laitière et de taux protéique (Coulon et Rémond, 1991), au moins dans le lot R. Dans le lot H, l'augmentation plus forte du taux protéique peut être due à l'amélioration plus importante des apports azotés à la sortie au pâturage. Elle ne peut en revanche pas être imputée à une amélioration de la qualité des apports azotés : les teneurs en méthionine et er, lysine des fourrages, dont on connaît l'effet spécifique sur le taux protéique du lait (Rulquin et al, 1993), n'ont en effet pas été différentes entre les périodes (tableau I).

Cette augmentation des apports nutritifs a pu être accentuée par le tri de l'herbe disponible qui permet aux animaux d'ingérer une herbe de meilleure valeur nutritive que ne le laisse supposer l'analyse d'échantillons prélevés manuellement, et/ou par une augmentation des quantités ingérées liée à une stimulation de l'appétit sous l'effet de facteurs climatiques, environnementaux ou sociaux. Enfin ces différents facteurs ont pu avoir un effet physiologique direct sur la répartition des nutriments entre la production de lait et de matières utiles et la constitution de réserves corporelles.
Les modifications importantes de la teneur en caséines du lait à la sortie au pâturage sont certainement la cause principale de l'augmentation de la fermeté du gel et de la diminution du temps de raffermissement, compte tenu de la forte liaison généralement observée entre ces paramètres (Remeuf et al, 1989 ; Vertès et al, 1989 ; Macheboeuf et al, 1993 ; Martin, 1993). Elles ont pu être amplifiées par la légère augmentation parallèle du taux de calcium du lait, parfois observée à la mise à l'herbe (Martin, 1993). Comme nous l'avions déjà mis en évidence (Macheboeuf et al, 1993), le pH du lait a augmenté à cette période et a conduit à une augmentation parallèle du temps de coagulation, comme cela est couramment observée dans d'autres situations (Remeuf et al, 1989 ; Martin et Coulon, 1991). Les raisons de cette augmentation restent difficiles à expliquer. Les facteurs de variation du $\mathrm{pH}$ du lait propres à l'animal restent en effet mal connus (Anderson, 1992).

En définitive, cette étude montre que l'effet favorable de la sortie au pâturage sur les performances des vaches laitières ne peut être attribué à la nature de la ration stricto sensu mais dépend essentiellement de la modification des apports nutritifs observés à cette période. Les causes de ces modifications (en particulier l'ingestibilité et/ou la valeur nutritive de l'herbe pâturée) ainsi que les effets directs ou indirects de facteurs non alimentaires (climatiques ou sociaux) sur les performances des animaux restent à confirmer.

\section{REMERCIEMENTS}

Nous tenons à remercier $S$ Bégon pour la réalisation des prélèvements et des mesures sur le lait, ainsi que $\mathrm{H}$ Rulquin et I Jicquel, qui ont réalisé la détermination de la composition en acides aminés des fourrages, et MF Mahé, qui a réalisé la détermination des variants génétiques des animaux. 


\section{RÉFÉRENCES}

Anderson RR (1992) Changes in milk pH and bicarbonate during 20 days of lactation in the guinea pig. $J$ Dairy Sci 75, 105-110

Chapman HR, Burnett $J$ (1972) Seasonal changes in the physical properties of milk for cheese making. Dainy Ind 37, 207-211

Coulon JB, Garel JP, Hoden A (1986) Évolution de la production et de la composition du lait à la mise à I'herbe. Bull Tech CRZV Theix INRA 66, 23-69

Coulon JB, D'Hour P. Petit M (1988) Influence of transition feeding pattern on milk production at the turn out of cows to pasture. Livest Prod Sci 20, 119-134

Coulon JB, Rémond B (1991) Variations in milk output and milk protein content in response to the level of energy supply to the dairy cow: a review. Livest Prod Sci $29,31-47$

Coulon JB, Faverdin P (1994) Vaches laitières. In : INRAtion, logiciel de calcul et d'analyse des rations pour les Ruminants. Version 2.5 (J Agabriel, P Champciaux, C Espinasse, eds), Cnerta, Dijon

Decaen C, Journet M (1966) Influence saisonnière sur la production et la composition du lait. Ann Zootech $15,259-277$

Decaen C, Ghadaki MB (1970) Variation de la sécrétion des acides gras des matières grasses du lait de vache à la mise à l'herbe et au cours des 6 premières semaines d'exploitation du fourrage vert. Ann Zootech 19, 399-411

Demarquilly C, Journet M (1962) Variations de la composition du lait à la mise à l'herbe. XVIe Congrès intern de laiterie, Copenhague, 33-78

Dubeuf B, Coulon JB, Landais E (1991) Mise à l'herbe des vaches laitières en zone de montagne: description des pratiques et liaison avec les performances laitières. INRA Prod Anim 4, 373-381

Grandison AS, Ford GD, Owen AJ, Millard D (1984) Chemical composition and coagulating properties of renneted friesian milk during the transition from winter rations to spring grazing. J Dairy Res $51,69-78$

Grosclaude $F(1988$ ) Le polymorphisme génétique des principales lactoprotéines bovines. Relations avec la quantité, la composition et les aptitudes fromagères du lait. INRA Prod Anim 1, 5-17

Hoden A, Coulon JB, Dulphy JP (1985) Influence de l'alimentation sur la composition du lait. III. Effets des régimes alimentaires sur les taux butyreux et protéiques. Bull Tech CRZV Theix INRA 62, 69-79

IOS (1987) Dried milk. Determination of sodium and potassium contents. Flame emission spectrophotometric method (ISO/DIS 8070)

Le Du YLP, Combellas J, Hodgson J, Baker RD (1979) Herbage intake and milk production by grazing dairy cows. II. The effects of level of winter feeding and daily herbage allowance. Grass For Sci 34, 249-260
Le Hénaff $L$ (1991) Importance des acides aminés dans la nutrition des vaches laitières. Thèse université de Rennes, $126 \mathrm{p}$

Machebœuf D, Coulon JB, D'Hour P (1993) Effect of breed, protein genetic variants and feeding on cows' milk coagulation properties. J Dairy Res 60, 43-54

Mariani P, Brizzi F, Pecorari M, Fossa E (1985) Le caratteristiche acidimetriche e di coagulazione del latte in rapporto al cambio primaverile di alimentazione. Sci Tec Latt Gas 36, 143-159

Martin B (1993) Influence des pratiques d'élevage et de fabrication fromagère sur les caractéristiques du lait et du fromage. Cas du reblochon fermier. Thèse ENSA, Montpellier, $82 p$

Martin B, Coulon JB (1991) Aptitude à la coagulation du lait de vache : influence de l'alimentation. Étude réalisée dans 6 exploitations du pays de Thônes (Haute-Savoie). INRA Prod Anim 4, 209-217

Mc Mahon DJ, Brown RJ (1982) Evaluation of formagraph for comparing rennet solutions. J Dairy $\mathrm{Sci}$ $65,1639-1642$

Moore JJ, Sax SM (1965) A revised automated procedure for urea nitrogen. Clin Chim Acta 11, 475-476

Rearte DH, Kesier EM, Hargrove GL (1986) Response by dairy cows to hay supplement with early spring grazing or to delay in turning out to pasture. $J$ Dairy Sci $69,1366-1373$

Remeuf F, Lenoir J, Duby C (1989) Étude des relations entre les caractéristiques physicochimiques des laits de chèvre et leur aptitude à la coagulation par la présure. Lait 71, 275-297

Rook JAF, Line C, Rowlands SJ (1960) The effect of the plane of energy nutrition of the cow during the late-winter feeding period on the changes in the solids-not-fat content of milk during the spring-grazing period. J Dairy Res 27, 427-433

Rowlands SJ (1938) The determination of the nitrogen distribution in milk. J Dairy Res 9, 42-46

Rulquin H, Pisulewski PM, Vérité R, Guinard J (1993) Milk production and composition as a function of postruminal lysine and methionine supply: A nutrientresponse approach. Livest Prod Sci 37, 69-90

Seibert B, Erhardt G, Senft B (1985) Procedure for simultaneous phenotyping of gentic variants in cow's milk by isoelectric focusing. Anim Blood Groups Bioch Gen 16, 183-191

Vérité $R$, Journet $M(1970)$ Influence de la teneur en eau et de la déshydratation de l'herbe sur sa valeur alimentaire pour les vaches laitières. Ann Zootech 19 , 255-268

Vertès C, Hoden A, Gallard Y (1989) Effet du niveau d'alimentation sur la composition chimique et la qualité fromagère du lait de vaches Holstein et Normandes. INRA Prod Anim 2, 89-96

Vignon B, Laurent $F$, Journet M (1978) Variations de la teneur en azote non protéique et en urée du lait à la mise à l'herbe des vaches laitières. Ann Zootech 27, 303-315 